Observati on of Transiti on froma

React i ve- Medi um I nst abi I i ty to an I nver se Landau Dampi ng in a Beam Pl asma System

\begin{tabular}{|l|l|}
\hline $\begin{array}{l}\text { j our nal or } \\
\text { publ i cat i on t i t l e }\end{array}$ & 福井大学工学部研究報告 \\
\hline vol une & 25 \\
\hline number & 1 \\
\hline page $r$ ange & $57-60$ \\
\hline year & $1977-03$ \\
\hline URL & ht t p: //hdl . handl e. net /10098/4477 \\
\hline
\end{tabular}


MEMO IRS OF THE FACULTY OF ENGINEER ING

FUKU I UNIVERS ITY

VOL. 25 No. 11977

\title{
Observation of Transition from a Reactive-Medium Instability to an Inverse Landau Damping in a Beam-Plasma System
}

\author{
Toshitaka IDEHARA; Nobuto MIYAMA, \\ Mataharu TANAKA * and Yoshio ISHIDA*
}

(Received Dec. 1, 1976)

\begin{abstract}
The wave excited by a coaxial probe in a beam-plasma system usually propagates as two waves which are independent of each other, i.e., a damped Trivelpiece mode of plasma and growing space charge wave of beam (refered to a reactive-medium instability). While, when the beam velocity is equal to or slightly langer than the phase velocity of plasma wave, the inverse Landau damping of the wave is observed, instead of the reactive-medium instability of beam wave.
\end{abstract}

The spatial growth of wave in a beam-plasma system has been investigated by many authors with a great interest. Two mechanisms of growing wave can be considered; One is the reactive medium instability of beam wave (two stream instability) and the other the inverse Landau damping of plasma wave. ${ }^{1}$ The former appears in the case where the modulation of beam applied spontaneously or artificially grows along the streaming of beam in a reactive plasma. Most instabilities which has been reported until now are considered to result from this mechanism. On the other hand, the latter can occur, only when the phase velocity of plasma wave coincides with the beam velocity and the modulation of beam is suppressed at lower level than that of plasma wave. Because the condition of the latter is more severe than that of the former, a growing wave refered to the latter is scarcely observed. In this letter, we report that the test wave excited by coaxial probe is usually grown due to

*) Department of Applied Physics 
the reactive medium instability, while the transition to the inverse Landau damping is observed under the severe condition for the plasma wave.

The plasma is produced by the dc discharge in the TP-D type device ${ }^{2}$ and diffused along the line of magnetic force into the chamber $(9.5 \mathrm{~cm}$ in diameter and $65 \mathrm{~cm}$ in length), its parameters being as follows. The density $n_{p}=$ $3.5 \sim 5.3 \times 10^{9} \mathrm{~cm}^{-3}$, the electron temperature $\mathrm{T}_{e}=7.0 \mathrm{eV}$, the magnetic field $\mathrm{B}=$ 180 gauss (homogeneity<3\%) corresponding to the electron cyclotron frequency $f_{c}\left(=\omega_{c} / 2 \pi\right)=504 \mathrm{MHz}$, the plasma diameter $D=30 \mathrm{~mm}$ and the pressure of the neutrals $(\mathrm{Ar}) \mathrm{p}=7.4 \times 10^{-4}$ torr (the collision frequency of electron with neutrals $u_{\text {en }}=4 \mathrm{MHz}$ is much smaller than $f_{c}$ ). The electron beam is generated by an electron gun placed at the opposite side of the discharge region and injected into the diffused plasma, so that the electron beam-plasma system is formed and the density $n_{b}$ and velocity $v_{b}$ (or energy $v_{b}$ ) of the beam are controlled independently of the plasma parameters. This device has been used in the experiment which was reported recently. $\left.{ }^{3}\right)$

The test wave is excited by the coaxial probe situated in the center of a beam-plasma system $(r=0$ and $z=0)$ and detected by the other antenna movable axially. By using the interferometer system, the propagating wave patterns along the field (along the axial direction $z$ ) are observed. They are shown in Fig. I ( $a$ ), with the exciting wave frequency $f$ as a parameter. It is seen in the figure that the slowly damping plasma wave (Trivelpiece mode) propagates near the exciting probe, while the wave growing along the streaming of beam does in the region far from the probe. The wave number $k_{\|}$for both waves are calculated by using these wave patterns and plotted as functions of wave frequency $f$ in Fig. $1(b)$, which shows the dispersion relation curves of the waves. As the latter wave satisfies the condition $\omega(=2 \pi f) \approx k_{11} v_{b}$, the growth is refered to the reactive-medium instability of the space charge wave of beam. ${ }^{4)}$ The dispersion relation curves intersect at the frequency $f=$ $280 \mathrm{MHz}$, where the beam velocity is equal to or slightly larger than the phase velocity of plasma wave. The wave pattern corresponding to this case is shown in the second trace of Fig. I (a). It is seen that the plasma wave propagates growing along the streaming of beam in the distance of a few wavelengths. The growth is refered to the inverse Landau damping of plasma wave. However, the mismatching between beam and phase velocities occurs and the plasma wave damps rapidly. In the region more far from the probe, the amplitude oscillation is observed.

The radial correlation of the wave is measured and the propagating wave surface is determined, which is shown in Fig. 2. Fig. 2 (a) shows the feature of waves in the case where the beam velocity is larger than the phase velocity 
of plasma wave. It is seen obviously that the wave excited near the probe (plasma wave) is quite different from that far from theprobe (beam wave). Fig. 2 (b) shows the case where the beam velocity is nearly equal to the phase velocity of plasma wave. The single wave does propagate along the streaming of beam in whole region.

In conclusion, the probe excites both plasma and beam waves in a beamplasma system, the former being a slowly damping wave near the probe and the latter appearing in the region far from the probe as growing wave refered to the reactive-medium instability. Especially, only when the beam velocity is nearly equal to phase velocity of plasma wave $\left(v_{b} \simeq \omega / k_{\|}\right)$, the plasma wave grows as the result of the inverse Landau damping.

References

1) For instance, Briggs; Electron-Stream Interaction with Plasmas, M.I.T. Press, Cambridge, Massachusetts (1964).

2) K. Takayama, M. Ohtsuka, Y. Tanaka, K. Ishii and Y. Kubota; in Proc. 7th Intern. Conf. on Phenomena in Ionized Gases (Gradevinska, Knjiga, Beograde, Yugoslavia, 1966) Vol. I, p.420.

3) T. Idehara, M. Takeda and Y. Ishida; Phys. Letters 58A (1976) 33.

4) T. Idehara, N. Miyama and Y. Ishida; J. Phys. Soc. Japan, to be published.

\section{Figure Captions}

Fig. I (a) Propagating wave patterns along the streaming of beam with the exciting frequency $f$ as a parameter. Beam voltage $v_{b}=120 \mathrm{~V}$. Normalized plasma density $\left(f_{p} / f_{c}\right)^{2}=0.5$ and normalized beam density $\left(f_{b} / f_{c}\right)^{2}=2 \times 10^{-3}$, where $f_{p}$ and $f_{b}$ are the plasma frequencies of plasma and beam. $\quad f_{c}=504 \mathrm{MHz}$.

(b) The dispersion relation of excited waves.

Fig. 2 Propagating wave surfaces. (a) the case of mismatching between beam and plasma wave and (b) the case of matching between beam and plasma wave. 


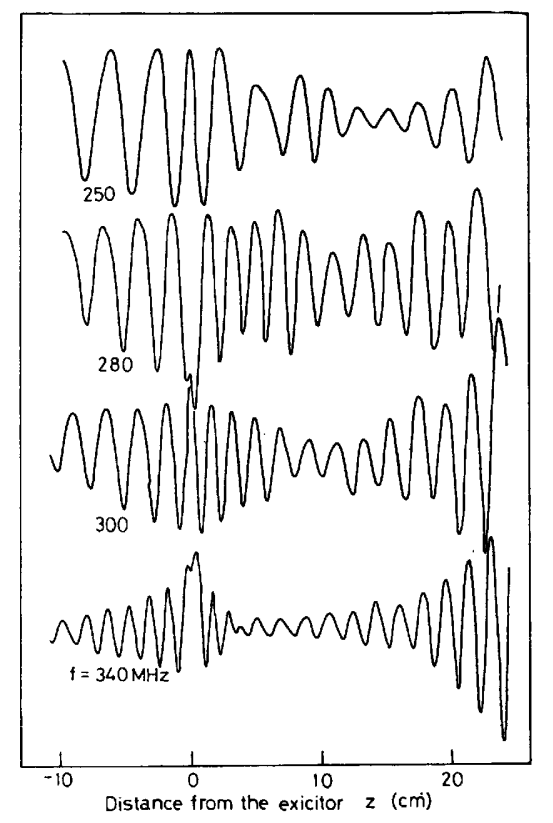

Fig. 1 (a)

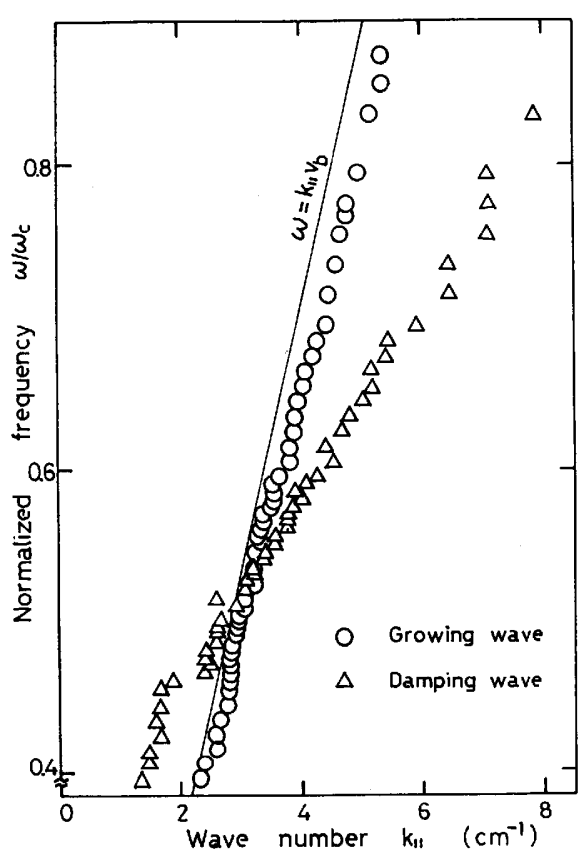

Fig. 1 (b)
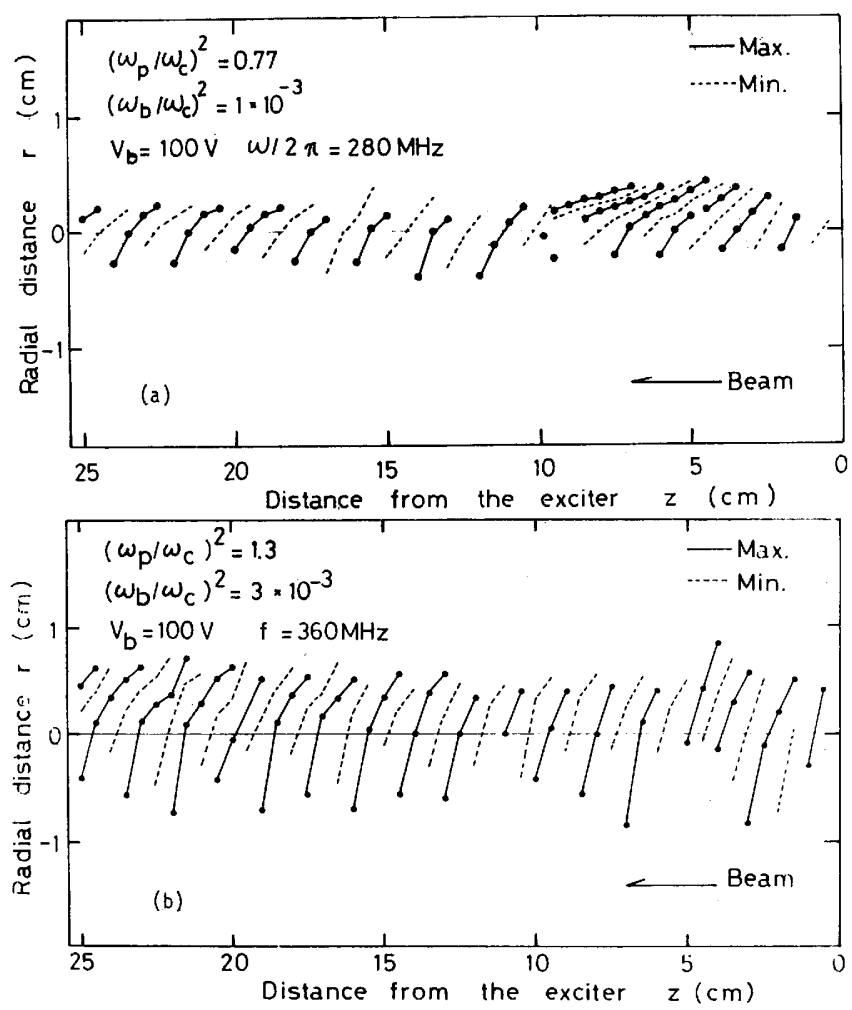

Fig. 2 